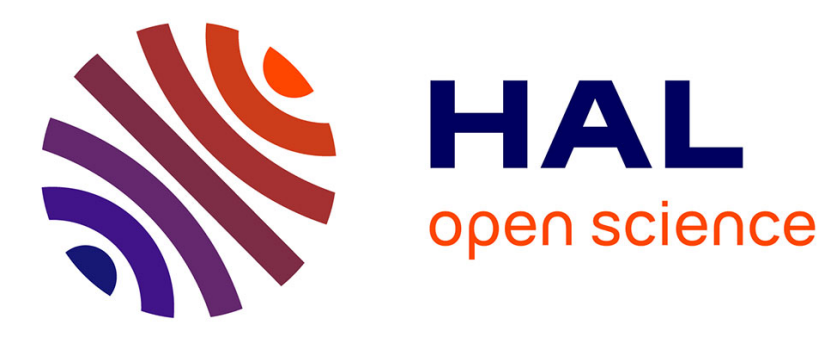

\title{
Bounded Delay and Concurrency for Earliest Query Answering
}

Olivier Gauwin, Joachim Niehren, Sophie Tison

\section{To cite this version:}

Olivier Gauwin, Joachim Niehren, Sophie Tison. Bounded Delay and Concurrency for Earliest Query Answering. 3rd International Conference on Language and Automata Theory and Applications, Apr 2009, Tarragona, Spain. pp.350-361, 10.1007/978-3-642-00982-2 . inria-00348463

\section{HAL Id: inria-00348463 https://hal.inria.fr/inria-00348463}

Submitted on 19 Dec 2008

HAL is a multi-disciplinary open access archive for the deposit and dissemination of scientific research documents, whether they are published or not. The documents may come from teaching and research institutions in France or abroad, or from public or private research centers.
L'archive ouverte pluridisciplinaire HAL, est destinée au dépôt et à la diffusion de documents scientifiques de niveau recherche, publiés ou non, émanant des établissements d'enseignement et de recherche français ou étrangers, des laboratoires publics ou privés. 


\title{
Bounded Delay and Concurrency for Earliest Query Answering
}

\author{
Olivier Gauwin ${ }^{123}$, Joachim Niehren ${ }^{13}$, and Sophie Tison ${ }^{23}$ \\ 1 INRIA, Lille \\ ${ }^{2}$ University of Lille 1 \\ 3 Mostrare project, INRIA \& LIFL (CNRS UMR8022)
}

\begin{abstract}
Earliest query answering is needed for streaming XML processing with optimal memory management. We study the feasibility of earliest query answering for node selection queries. Tractable queries are distinguished by a bounded number of concurrently alive answer candidates at every time point, and a bounded delay for node selection. We show that both properties are decidable in polynomial time for queries defined by deterministic automata for unranked trees. Our results are obtained by reduction to the bounded valuedness problem for recognizable relations between unranked trees.
\end{abstract}

\section{Introduction}

Streaming algorithms are relevant for XML databases and data exchange, whenever large data collections are to be processed that cannot be stored in main memory. Instead data is communicated over streams and processed incrementally. Recently, XML streaming algorithms were proposed for schema validation [1] (membership in tree languages), one-pass typing [2] (annotating nodes of trees by types), and query answering [3-5].

The space complexity of streaming algorithms for answering node selection queries in XML trees depends on the size of the call stack (bounded by the depth of the tree) and on the number of concurrently alive answer candidates that are kept in main memory at every time point [6]. The purpose of earliest query answering (EQA) is to minimize the second number. Selection and unselection of answer candidates needs to be decided as early as possible, so that selected candidates can be output and unselected candidates discarded as early as possible. In both cases they are removed from main memory.

EQA is the objective of various streaming algorithms for Forward XPath and its fragments [6-8], and has been studied for automata defined queries too [9, 10]. In the latter paper, it is shown how to obtain a correct EQA algorithm for a fragment of Forward XPath under schema assumptions, by a P-time translation to deterministic streaming tree automata (dSTAs) [11], or equivalently deterministic nested word [12], visibly pushdown [13] or pushdown forest automata [14].

Whether EQA is tractable depends on two properties of the considered query $Q$ and schema $S$, both of which are independent of the concrete algorithm. The 
first restriction is bounded delay of selection, which requires a bound for all trees $t \in S$ on the number of events between the first visit of a selected node $\pi \in Q(t)$ and the earliest event that permits its selection. This limits the waiting time for the answer. The second restriction is bounded concurrency of alive candidates, which imposes a bound on the number of concurrently alive answer candidates for $Q$ wrt. $S$ for all trees of $S$ at all time points. This limits the maximal number of candidates that need to be memoized simultaneously by every EQA algorithm.

In this paper, we show that bounded delay and bounded concurrency are decidable in P-time for queries and schemas defined by deterministic automata for unranked trees. Our result holds for dSTAs, as well as for bottom-up deterministic tree automata that operate on binary encodings of unranked trees [15], either firstchild-nextsibling based or Curried [16]. When restricting databases to words instead of trees, decision procedures for bounded delay and concurrency for queries defined by dFAs can be obtained quite easily by reduction to bounded ambiguity of nFAs. The algorithm for words, however, cannot be lifted to trees in any straightforward manner. In order to solve this problem, we propose another solution by reduction to the bounded valuedness problem of recognizable relations between unranked trees. As we show, this problem can be reduced to bounded valuedness of bottom-up tree transducers, which can be decided in Ptime (Theorem 2.8 of [17]). All reductions are in P-time since we start from deterministic automata. Omitted proofs can be found in the long version.

\section{Queries in Words}

We recall definitions of schemas and queries for tuples of positions in words by dFAs, and the concept of bounded ambiguity for nFAs.

Words, Positions, and Events. Let an alphabet $\Sigma$ be a finite set with elements ranged over by $a, b, c$ and $\mathbb{N}$ the set of natural numbers $n \geq 1$. We will consider words $w \in \Sigma^{+}$as databases. The set of all words $\Sigma^{*}$ is closed under concatenation $w w^{\prime}$ and contains the empty word $\epsilon$. The set of positions of a word $w \in \Sigma^{*}$ is $\operatorname{pos}(w)=\{1, \ldots,|w|\}$ where $|w|$ is the number of letters of $w$. For all $w \in \Sigma^{+}$and positions $\pi \in \operatorname{pos}(w)$ we define $l a b^{w}(\pi) \in \Sigma$ to be the $\pi$-th letter of word $w$ and say that position $\pi$ is labeled by $l a b^{w}(\pi)$.

One-way finite automata process words letter by letter from the left to the right, equally to streaming algorithms for words. We define the set of events for a word $w \in \Sigma^{+}$by adding the start event 0 to the set of all positions eve $(w)=\{0\} \cup \operatorname{pos}(w)$. For all events $e \in e v e(w)$, we define $w \leq e \in \Sigma^{*}$ to be the prefix of $w$ with exactly $e$ letters. We say that two words coincide until event $e$ if $w^{\leq e}=w^{\prime \leq e}$ and write $e q_{e}\left(w, w^{\prime}\right)$ in this case.

Queries and Schemas. An $n$-ary query $Q$ selects a set of $n$-tuples of positions for every word $w \in \Sigma^{+}$. It is a function which maps words $w$ to sets of tuples of positions $Q(w) \subseteq \operatorname{pos}(w)^{n}$. A schema $S \subseteq \Sigma^{+}$restricts the set of permitted databases. We say that a word $w \in \Sigma^{+}$satisfies a schema $S$ if and only if $w \in S$.

Automata, Ambiguity, and Determinism. A finite automaton (nFA) over $\Sigma$ is a tuple $A=($ stat, init, rul, fin) where init, fin $\subseteq$ stat are finite sets and 
$r u l \subseteq$ stat $^{2} \times(\Sigma \cup\{\epsilon\})$ contains rules that we write as $q \stackrel{a}{\rightarrow} q^{\prime}$ or $q \stackrel{\epsilon}{\rightarrow} q^{\prime}$ where $q, q^{\prime} \in$ stat and $a \in \Sigma$. Whenever necessary, we will index the components of $A$ by $A$. Let the size of $A$ count all states and rules, i.e., $|A|=\left|s t^{2} t_{A}\right|+\left|r u l_{A}\right|$.

A run of $A$ on a word $w$ is a function $r: \operatorname{eve}(w) \rightarrow \operatorname{stat}_{A}$ so that $r(0) \in$ init $_{A}$ and $r(\pi-1) \stackrel{\epsilon * a}{\rightarrow} \stackrel{\epsilon}{\rightarrow} r(\pi)$ is justified by $r u l$ for all $\pi \in \operatorname{pos}(w)$ with $a=l a b^{w}(\pi)$. A run is successful if $r(|w|) \in f i n_{A}$. The language $L(A) \subseteq \Sigma^{*}$ is the set of all words that permit a successful run by $A$. An nFA is called productive, if all its states are used in some successful run. This is the case if all states are reachable from some initial state, and if for all states, some final state can be reached.

The ambiguity $a m b_{A}(w)$ is the number of successful runs of $A$ on $w$. The ambiguity of $A$ is $k$-bounded if $a m b_{A}(w) \leq k$ for all $w \in \Sigma^{*}$. It is bounded, if it is bounded by some $k$. An nFA is deterministic or a dFA if it has at most one initial state and at most one rule for all left hand sides, including letters. Clearly the ambiguity of dFAs is 1-bounded.

Stearns and Hunt [18] present a P-time algorithm for deciding $k$-bounded ambiguity of nFAs. Let us write $p \stackrel{w}{\rightarrow} q$ by $A$ if there exists a run of $A[$ init $=\{p\}]$ (the automaton obtained from $A$ by setting its initial states to $\{p\}$ ) on $w$ that ends in $q$. Weber and Seidl [19] show that an nFA $A$ has unbounded ambiguity iff there exists a word $w \in \Sigma^{+}$and distinct states $p \neq q$ such that $p \stackrel{w}{\rightarrow} p, p \stackrel{w}{\rightarrow} q$, $q \stackrel{w}{\rightarrow} q$ by $A$. This can be tested in $O\left(|A|^{3}\right)$ as shown very recently by [20].

Canonicity and Types. Let $\mathbb{B}=\{0,1\}$ be the set of Booleans. For words $w \in \Sigma^{*}$ and tuples $\tau=\left(\pi_{1}, \ldots, \pi_{n}\right) \in \operatorname{pos}(w)^{n}$, let $w * \tau$ be the annotated word in $\left(\Sigma \times \mathbb{B}^{n}\right)^{*}$ obtained from $w$ by relabeling all positions $\pi \in \operatorname{pos}(w)$ to $\left(l a b^{w}(\pi), b_{1}, \ldots, b_{n}\right)$, where $b_{i}=1$ if $\pi=\pi_{i}$ and $b_{i}=0$ otherwise. The canonical language of an n-ary query $Q$ is $\operatorname{can}_{Q}=\{w * \tau \mid \tau \in Q(w)\}$. The type of a word $v \in\left(\mathbb{B}^{n}\right)^{*}$ is an element of $(\mathbb{N} \cup\{0\})^{n}$ defined by $\sum_{\pi \in \operatorname{pos}(v)} l a b^{v}(\pi)$. The type of a word in $\left(\Sigma \times \mathbb{B}^{n}\right)^{*}$ is the type of its projection in $\left(\mathbb{B}^{n}\right)^{*}$. All words over $\Sigma \times \mathbb{B}^{n}$ of type $1^{n}$ have the form $w * \tau$, and vice versa. An nFA $A$ over $\Sigma \times \mathbb{B}^{n}$ is called canonical if all words of $L(A)$ have type $1^{n}$. For productive canonical dFAs $A$, every state $q \in$ stat has a unique type in $\mathbb{B}^{n}$, which is the type of all words with runs by $A$ ending in $q$ (e.g., Lemma 3 of [21]).

\section{Earliest Query Answering for Words}

We recall the framework of EQA for XML databases from [10], but restricted to the case of words, and show for queries defined by dFAs that bounded concurrency and delay can be reduced in P-time to bounded ambiguity for dFAs.

An EQA algorithm decides selection and failure of answer candidates at every time point (without knowing the rest of the stream). This way, it needs to keep in main memory only alive candidates, which are neither safe for selection nor failure. As an example, consider the monadic query $Q$ that selects all positions in words $w$ that are labeled by $a$ and followed by $b b$. When applied to $w=$ $a a b b a b b c a b a b$, this query returns $Q(w)=\{(2),(5)\}$. A streaming algorithm can enumerate these answers by using a sliding window of length 3. Position 1 for 
instance can be refused when having seen the labels of positions 1,2 , while position 2 can be selected when having seen the labels of positions $2,3,4$.

Schema assumptions are relevant to EQA since restricting the remainder of the stream. The schema $(a \mid b)^{*} c(a b)^{*}$, for instance, excludes all positions from $Q(w)$ that are on the right of the $c$ letter in $w$. This allows to exclude positions $8, \ldots, 12$ to belong to the answer set $Q(w)$ immediately at the respective position.

Earliest Selection and Bounded Delay. The delay of a selected position is the number of subsequent events before selection can be safely decided. More formally, let $Q$ be an $n$-ary query in words $w \in \Sigma^{+}$satisfying a schema $S \subseteq \Sigma^{+}$. We define a relation $\operatorname{sel}_{Q}^{S}(w)$ that links tuples $\tau \in \operatorname{pos}(w)^{n}$ to events $e \in \operatorname{eve}(w)$ that are sufficient for selection, i.e., where $\tau$ will be selected in all possible continuations of the stream beyond $e$. Only those continuations are allowed, which extend the current prefix of the word to a member of $S$ :

$$
(\tau, e) \in \operatorname{sel}_{Q}^{S}(w) \Leftrightarrow \tau \in\{1, \ldots, e\}^{n} \wedge \forall w^{\prime} \in S . e q_{e}\left(w, w^{\prime}\right) \Rightarrow \tau \in Q\left(w^{\prime}\right)
$$

Note that the initial event 0 may be sufficient to select the empty tuple () in Boolean queries where $n=0$, while it is never sufficient for selection if $n \geq 1$ since otherwise $\tau \notin\{1, \ldots, e\}^{n}$.

Let latest $\left(\left(\pi_{1}, \ldots, \pi_{n}\right)\right)=\max _{1 \leq i \leq n} \pi_{i}$ be the latest position of the tuple. The delay of an $n$-ary query $Q$ for a tuple $\tau \in \operatorname{pos}(w)$ is the number of events $e$ following latest $(\tau)$ such that $e$ is insufficient for selection, i.e., $(\tau, e) \notin \operatorname{sel}_{Q}^{S}(w)$.

$$
\operatorname{delay}_{Q}^{S}(w, \tau)=\left|\left\{e \in \operatorname{eve}(w) \mid \operatorname{latest}(\tau) \leq e,(\tau, e) \notin \operatorname{sel}_{Q}^{S}(w)\right\}\right|
$$

Query $Q$ with schema $S$ has $k$-bounded delay if $\operatorname{delay}_{Q}^{S}(w, \tau) \leq k$ for all $w \in S$ and $\tau \in Q(w)$. It has bounded delay if it has $k$-bounded delay for some $k \geq 0$. Having bounded delay means that every EQA algorithm will output selected tuples a constant time after completion.

Deciding Bounded Delay. We start with the case without schemas. Let $A$ be a canonical and productive dFA over $\Sigma \times \mathbb{B}^{n}$ and $Q_{A}$ the $n$-ary query that it defines. We call a state $q \in$ stat $_{A}$ of type $1^{n}$ safe for selection if $\left(\Sigma \times\{0\}^{n}\right)^{*} \subseteq$ $L(A[$ init $=\{q\}])$. Since $A$ is canonical and deterministic, this is the case if and only if all states reachable from $q$ are final and have outgoing transitions for all letters in $\Sigma \times\{0\}^{n}$. Thus, the set of states of $A$ that are safe for selection can be computed in time $O(|A|+|\Sigma|+n)$.

Lemma 1. If the run $r$ of a canonical dFA $A$ on $w * \tau$ exists then it satisfies for all $e \in \operatorname{pos}(w)$ that $r(e)$ is safe for selection if and only if $(\tau, e) \in \operatorname{sel}_{Q_{A}}(w)$.

We define an nFA $D(A)$ such that $a m b_{D(A)}(w * \tau)=\operatorname{delay}_{Q_{A}}(w, \tau)$ for all $\tau \in Q_{A}(w) . D(A)$ has the same states as $A$ except for one additional state $o k$, which is the only final state of $D(A)$. All transitions of $A$ are preserved by $D(A)$. In addition to simulating $A$, automaton $D(A)$ has $\epsilon$ transitions into the state $o k$ from all states $q$ of type $1^{n}$ of $A$ that are unsafe for selection. State $o k$ has transitions into itself for all letters in $\Sigma \times\{0\}^{n}$.

Proposition 1. For $w \in \Sigma^{*}, \tau \in Q_{A}(w): \operatorname{delay}_{Q_{A}}(w, \tau)=\operatorname{amb}_{D(A)}(w * \tau)$. 
Proof. Consider a run of $D(A)$ on a canonical word $w * \tau$. The only ambiguity of $D(A)$ is introduced by the $\epsilon$-transitions, by which to exit from $A$ at positions between the last component of $\tau$ (included) and the earliest event that is safe for the selection of $\tau$. The number of such positions is precisely $\operatorname{delay}_{Q_{A}}(w, \tau)$.

Theorem 1. Bounded delay for queries $Q_{A}$ and schemas $L(B)$ defined by dFAs $A, B$ can be decided in time $O(|A| \cdot|B|)$, and $k$-bounded delay in P-time.

Proof. We sketch the proof for bounded delay without schemas, where $L(B)=$ $\Sigma^{*}$. By Proposition 1, it is sufficient to decide whether $D(A)$ has bounded ambiguity. By Weber and Seidl's characterization, this holds if the subautomaton of $A$ containing only unsafe states for selection of type $1^{n}$, has no loop. Acyclicity of this subautomaton can be tested in time $O(|A|)$.

Earliest Failure and Bounded Concurrency. The space complexity of EQA algorithms depends on the concurrency of a query, which is the maximal number of concurrently alive answer candidates at every time point $[6,7]$, since these are to be kept in main memory. In order to formalize this for $n$-ary queries, we have to deal with partial answer candidates for a given word $w$. We fix a constant - that represents unknown components, and define partial tuples $\tau$ of positions until $e \in \operatorname{pos}(w)$ as members of $(\{1, \ldots, e\} \uplus\{\bullet\})^{n}$. So far, we have only studied complete answer candidates, which do not contain any unknown component. We write $\operatorname{compl}(\tau, w, e)$ for the set of complete candidates, in which all unknown components of $\tau$ have been instantiated with nodes $\pi \in \operatorname{pos}(w)$ such that $e \leq \pi$. Given a query $Q$, schema $S$, and word $w \in S$, we call a partial candidate $\tau$ failed at event $e \in \operatorname{eve}(w)$, if no completion of $\tau$ by nodes in the future of $e$ is selected by $Q$.

$$
(\tau, e) \in \operatorname{fail}_{Q}^{S}(w) \Leftrightarrow\left\{\begin{array}{l}
\tau \in(\{1, \ldots, e\} \uplus\{\bullet\})^{n} \wedge \\
\forall w^{\prime} \in S . e q_{e}\left(w, w^{\prime}\right) \Rightarrow \forall \tau^{\prime} \in \operatorname{compl}\left(\tau, w^{\prime}, e\right) . \tau^{\prime} \notin Q\left(w^{\prime}\right)
\end{array}\right.
$$

A partial candidate $\tau \in(\{1, \ldots, e\} \cup\{\bullet\})^{n}$ is alive at $e$ if it is neither failed nor selected at $e$. The concurrency of a query schema pair on a word $w \in \Sigma^{+}$at position $e \in \operatorname{eve}(w)$ is the number of alive candidates at time point $e$, so that $e$ is neither sufficient for selection or failure:

$$
\begin{aligned}
& (\tau, e) \in \operatorname{alive}_{Q}^{S}(w) \Leftrightarrow(\tau, e) \notin \operatorname{fail}_{Q}^{S}(w) \text { and }(\tau, e) \notin \operatorname{sel}_{Q}^{S}(w) \\
& \operatorname{concur}_{Q}^{S}(w, e)=\left|\left\{\tau \in(\{1, \ldots, e\} \cup\{\bullet\})^{n} \quad \mid(\tau, e) \in \operatorname{alive}_{Q}^{S}(w)\right\}\right|
\end{aligned}
$$

We say that the concurrency of a query schema pair is bounded if there exists $k \geq 0$ such that $\operatorname{concur}_{Q}^{S}(w, e) \leq k$ for all words $w \in S$ and $e \in \operatorname{pos}(w)$.

Deciding Bounded Concurrency. We start with queries without schemas. So let $A$ be a canonical productive dFA over $\Sigma \times \mathbb{B}^{n}$ and $Q_{A}$ the query it defines. Recall that all states of $A$ have a unique type $v \in \mathbb{B}^{n}$. We call a state $q$ of type $v$ safe for failure, if no final states can be reached from $q$ by words of complementary type $1^{n}-v$. By canonicity, this is the case if no final states can be reached from $q$ at all. We can thus compute the set of safe states for failure in time $O(|A|)$. 
Lemma 2. If the unique run $r$ of a canonical dFA $A$ on some $w * \tau$ exists, then all $e \in \operatorname{pos}(w)$ satisfy that $r(e)$ is safe for failure if and only if $(\tau, e) \in$ fail $_{Q_{A}}(w)$.

We define an nFA $C(A)$ such that $a m b_{C(A)}(w * e)=\operatorname{concur}_{Q}(w, e)$ for all $e \in \operatorname{pos}(w)$. The situation is a little different than for $D(A)$ since $C(A)$ runs on words annotated by events rather than tuples. So the alphabet of $C(A)$ is $\Sigma \times \mathbb{B}$. The nFA $C(A)$ guesses all partial candidates with positions until $e$, tests whether they are alive at $e$, and accepts in this case and only this case.

Proposition 2. For all $e \in \operatorname{pos}(w): \operatorname{concur}_{Q_{A}}(w, e)=\operatorname{amb}_{C(A)}(w * e)$.

Theorem 2. Bounded and $k$-bounded concurrency for queries and schemas defined by canonical dFAs can be decided in P-time for any fixed $k \geq 0$.

\section{$4 \quad$ Earliest Query Answering for Unranked Trees}

We extend EQA from words to unranked trees. We then lift our P-time decision results to tree automata for unranked trees, and argue why the proofs for words cannot be lifted in any straightforward manner.

The set of unranked trees $T_{\Sigma}$ is the least set that contains all $(\mathrm{k}+1)$-tuples $a\left(t_{1}, \ldots, t_{k}\right)$ where $k \geq 0, a \in \Sigma$ and $t_{1}, \ldots, t_{k} \in T_{\Sigma}$. Positions of words correspond to nodes of trees, defined by $\operatorname{nod}\left(a\left(t_{1}, \ldots, t_{k}\right)\right)=\{\epsilon\} \cup\left\{i \pi \mid \pi \in \operatorname{nod}\left(t_{i}\right)\right\}$. The word $w=a b a c a$, for instance, can be encoded by the tree $t=r(a, b, a, c, a)$, where $r \in \Sigma$ is an arbitrary symbol. Note that $\operatorname{nod}(t)=\{\epsilon\} \cup \operatorname{pos}(w)$. Queries $Q$ in unranked trees select tuples of nodes $Q(t) \subseteq \operatorname{nod}(t)^{n}$ for all trees $t \in T_{\Sigma}$. Events are produced by preorder traversals:

$$
\operatorname{eve}(t)=\{\text { start }\} \cup(\{\text { open, } \text { close }\} \times \operatorname{nod}(t))
$$

There is an initial event start and an opening and a closing event per node. Let $\leq$ be the total order on eve $(t)$ induced by preorder traversals over trees $t \in T_{\Sigma}$, and let $\operatorname{pred}(e)$ be the immediate predecessor of event $e \in$ eve $(t)-\{$ start $\}$. For all $e \in e v e(t)-\{$ start $\}$, we define the prefix $t^{\leq e}$ of $t$ to be the tree which contains the part of $t$ with all nodes opened before e, i.e., $\operatorname{nod}\left(t^{\leq e}\right)=\{\pi \in$ $\operatorname{nod}(t) \mid$ (open, $\pi) \leq e\}$, and $l a b^{t^{\leq e}}(\pi)=\operatorname{lab} b^{t}(\pi)$ for all $\pi \in \operatorname{nod}\left(t^{\leq e}\right)$. Note that $t \leq(\operatorname{close}, \pi)$ contains all proper descendants of $\pi$ in $t$, while $t \leq$ (open, $\pi)$ does not. As before, we can define $e q_{e}\left(t, t^{\prime}\right)$ by $e=$ start or $t^{\leq e}=t^{\prime \leq e}$. The notion $t * \tau \in T_{\Sigma \times \mathbb{B}^{n}}$ extends straightforwardly from words to trees. The canonical language of an $n$-ary query $Q$ thus has type $\operatorname{can}_{Q} \subseteq T_{\Sigma \times \mathbb{B}^{n}}$. The definitions of $\operatorname{sel}_{Q}^{S}$, fail $_{Q}^{S}$, delay $_{Q}^{S}$ and concur ${ }_{Q}^{S}$ extend literally, except that the set $\{1, \ldots, e\}$ needs to be replaced by $\operatorname{nod}(t \leq e)$.

Tree automata for unranked trees are often obtained from standard tree automata for binary trees. A binary signature is a finite set $\Gamma=\Gamma_{2} \uplus \Gamma_{0}$ with constants in $\Gamma_{0}$ and binary function symbols in $\Gamma_{2}$. The set of binary trees $T_{\Gamma}^{\text {bin }}$ is the least set containing all $c \in \Gamma_{0}$ and triples $f\left(t_{1}, t_{2}\right)$ where $f \in \Gamma_{2}$ and $t_{1}, t_{2} \in$ $T_{\Gamma}^{\mathrm{bin}}$. A tree automaton (TA) for binary trees in $T_{\Gamma}^{\mathrm{bin}}$ is a tuple $A=($ stat, fin, $\mathrm{rul})$ 


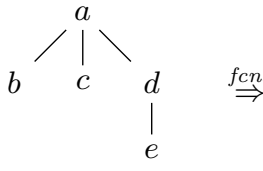

Fig. 1.

Binary encoding
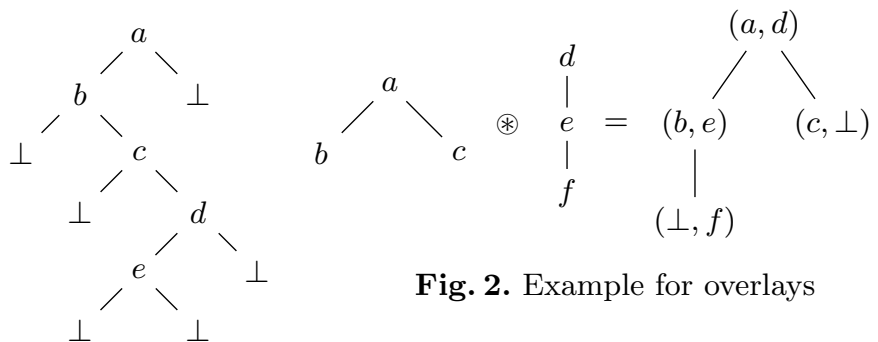

Fig. 2. Example for overlays

consisting of finite sets fin $\subseteq$ stat and a set $r u l \subseteq \cup_{i \in\{0,2\}}$ stat $^{i+1} \times \Gamma_{i}$, that we denote as $f\left(q_{1}, q_{2}\right) \rightarrow q$ and $c \rightarrow q$ where $q_{1}, q_{2}, q \in$ stat, $f \in \Gamma_{2}$ and $c \in \Gamma_{0}$. A run of $A$ on $t \in T_{\Gamma}^{\text {bin }}$ is a function $r: \operatorname{nod}(t) \rightarrow$ stat such that $f(r(\pi 1), r(\pi 2)) \rightarrow r(\pi)$ belongs to $r u l_{A}$ for all nodes $\pi$ of $t$ with $l a b^{t}(\pi)=f \in \Gamma_{2}$, and $r(\pi) \rightarrow c$ in $r u l_{A}$ for all nodes $\pi$ of $t$ with $l a b^{t}(\pi)=c \in \Gamma_{0}$. The language $L^{\text {bin }}(A)$ is the set of all binary trees over $\Gamma$ that permit an successful run by $A$, where $r(\epsilon) \in \operatorname{fin}$. A (bottom-up) deterministic TA (dTA) is a TA of which no two rules have the same left hand side.

We can encode unranked trees $t \in T_{\Sigma}$ into binary trees by applying Rabin's firstchild-nextsibling encoding fcns $: T_{\Sigma} \rightarrow T_{\Sigma \perp}^{\text {bin }}$ where $\Sigma_{\perp}=\Sigma \uplus\{\perp\}$. The definition is recalled by example in Fig. 1 . A TA over $T_{\Sigma_{\perp}}$ defines the language of unranked trees $L(A)=\left\{t \in T_{\Sigma} \mid f \operatorname{cns}(t) \in L^{\text {bin }}(A)\right\}$.

Operationally, however, dTAs fail to operate in streaming manner on unranked trees, so that the previous decision algorithms cannot be lifted to queries defined by dTAs. Streaming tree automata (STAs) [11] operate in the proper order. They are a reformulation of nested word automata $[12,13]$ and shown equivalent to pushdown forest automata [14]. Deterministic STAs (dSTAs) can perform one-pass typing for extended DTDs with restrained competition [2] as well as EQA [10] for queries defined by dSTAs. Furthermore, deterministic stepwise tree automata [16] can be converted in dSTAs in linear time.

Proposition 3 (Closure properties). The classes of TAs (wrt. the fcns encoding) and STAs permit determinization, and recognize the same langages of unranked trees modulo P-time automata translations (not preserving determinism). Recognizable languages are closed under Boolean operations, projection and cylindrification. All corresponding operations on TAs (resp. STAs) can be performed in P-time and preserve determinism except for projection.

Even with STAs, it remains difficult to lift our P-time algorithms for words to trees, since the notion of safe states becomes more complex. Given a canonical dSTA $A$ for query $Q_{A}$, one can define another dSTA $E(A)$ for which appropriate notions of safe states wrt. $Q_{A}$ exist [10]. The size of $E(A)$, however, may grow exponentially in $|A|$. Therefore, we cannot use $E(A)$ to construct polynomially sized counterparts of $D(A)$ and $C(A)$ in the case of unranked trees. Nevertheless:

Theorem 3 (Main). Bounded delay is decidable in P-time for n-ary queries and schemas in unranked trees defined by dTAs (wrt. the fcns or Curried en- 


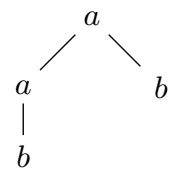

(a) Tree $t$

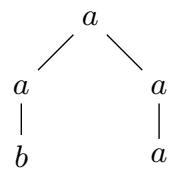

(b) Tree $s$

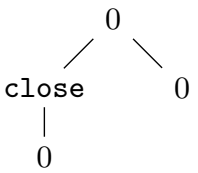

(c) Tree $s_{e}$

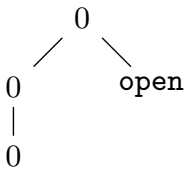

(d) Tree $s_{e^{\prime}}$

Fig. 3. $\left(t, s, s_{e}\right) \in E q$ but $\left(t, s, s_{e^{\prime}}\right) \notin E q$

coding) or dSTAs, where $n$ may be variable. Bounded concurrency is decidable in P-time for fixed $n$. For fixed $k$ and $n, k$-bounded delay and concurrency are decidable in NP-time.

Our proof will be based on the powerful notion of recognizable relations between unranked trees (see [15] for ranked trees). Bounded delay and concurrency are reduced to bounded valuedness of recognizable relations, which in turn is reduced to bounded valuedness of tree transducers for binary trees [17].

\section{Recognizable Relations between Unranked Trees}

We extend the theory of recognizable relations from ranked to unranked trees. We show that FO-formulas over recognizable relations with $n$ free variables define recognizable relations between $n$ unranked trees (so that satisfiability is decidable), and that bounded valuedness of recognizable relations can be decided in P-time by reduction to bounded valuedness of tree transducers (for binary trees).

Recognizable Relations. In this section, we assume an arbitrary class of tree automata, that satisfy the properties of STAs in Proposition 3. This includes TAs modulo the fcns encoding, STAs, and stepwise tree automata [16] (but not deterministic hedge automata with dFAs for horizontal languages [15]).

The overlay of $k$ unranked trees $t_{i} \in T_{\Sigma^{i}}$ is the unranked tree $t_{1} \circledast \ldots \circledast t_{k}$ in $T_{\Sigma_{\perp}^{1} \times \ldots \times \Sigma^{k}}$ obtained by superposing these $k$ trees top-down and left-to-right; the $\perp$ symbol represent missing children where the structures of the trees differ. This is illustrated in Fig. 2. Overlays of ranked trees can be obtained this way too [15], except that overlayed symbols need to inherit the maximal arity. A $k$-ary relation $R$ between unranked trees is recognizable iff the language of its overlays $\operatorname{ovl}(R)=\left\{t_{1} \circledast \ldots \circledast t_{k} \mid\left(t_{1}, \ldots, t_{k}\right) \in R\right\}$ is recognizable by a tree automaton. We say that $R$ is recognized by the automaton $A$ if $\operatorname{ovl}(R)=L(A)$. We also say that $R$ can be computed in time $k$ if an automaton recognizing $R$ can be computed in time $k$.

The prime example is the relation $E q \subseteq T_{\Sigma} \times T_{\Sigma} \times T_{\{0, \text { open,close }\}}$. Here, we map event $e=(\alpha, \pi) \in$ eve $(t)$ to trees $\operatorname{ren}^{e}(t) \in T_{\{0, \text { open,close }\}}$ obtained by relabeling $t$, such that $\pi$ is relabeled to $\alpha$ and all other nodes to 0 . We then define $E q=\left\{\left(t, s, \operatorname{ren}^{e}(t)\right) \mid e q_{e}(t, s)\right\}$. See Fig. 3 for an example.

Lemma 3. Given a signature $\Sigma$, a deterministic automaton recognizing relation $E q \subseteq T_{\Sigma} \times T_{\Sigma} \times T_{\{0, \text { open, close }\}}$ can be computed in time $O\left(|\Sigma|^{2}\right)$. 
An STA recognizing $\operatorname{ovl}(E q)$ with $O(1)$ states is easy to define. It can be converted into a TA by Proposition 3 and from there to a deterministic automaton of the class under consideration by assumption. The resulting automaton still has $O(1)$ states, and thus an overall size of $O(|\Sigma|)$, if we assume in addition a function $\psi$ such that $|A| \leq|\Sigma| \cdot \psi\left(\mid\right.$ stat $\left._{A} \mid\right)$ for all automata $A$ with signature $\Sigma$.

FO Logic. Let $\Omega$ be a collection of unranked disjoint signatures and $\Re$ a set of recognizable relations between unranked trees, so that each relation $R \in \Re$ has a type $R \subseteq T_{\Sigma_{1}^{R}} \times \ldots \times T_{\Sigma_{a r(R)}^{R}}$ where $\Sigma_{1}^{R}, \ldots, \Sigma_{\operatorname{ar}(R)}^{R} \in \Omega$ and $\operatorname{ar}(R) \geq 0$. We fix an infinite set of variables $V$ ranging over unranked trees. A FO formula over recognizable relations in $\Re$ and signatures in $\Omega$ has the abstract syntax:

$$
\phi::=R\left(X_{1}, \ldots, X_{\operatorname{ar}(R)}\right)\left|\phi \wedge \phi^{\prime}\right| \neg \phi \mid \exists X \in T_{\Sigma} \cdot \phi
$$

where $R \in \Re, X_{1}, \ldots, X_{a r(R)} \in V$, and $\Sigma \in \Omega$. We assume that all formulas are well-typed, i.e., that the types of variables are compatible with those of the relations in which they appear. A formula $\phi$ with $m$ free variables $X_{1}, \ldots, X_{m}$ of types $T_{\Sigma_{1}}, \ldots, T_{\Sigma_{m}}$ defines an $m$-ary relation $R_{\phi\left(X_{1}, \ldots, X_{m}\right)} \subseteq T_{\Sigma_{1}} \times \ldots \times T_{\Sigma_{m}}$. The closure properties of tree automata ensure that all such relations are recognizable. Let $F O_{\exists}[\Re]$ be the set of existential formulas where existential quantifier are restricted to occur outermost (and $\Omega$ is the set of signatures appearing in the types of relations in $\Re$ ). The size $|\phi|$ is the number of nodes of $\phi$.

Proposition 4. Let $\Re$ be a finite set of relations recognized by deterministic

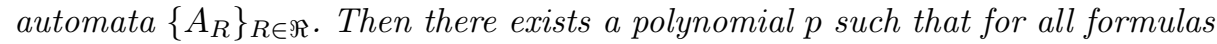
$\phi$ in $\mathrm{FO}_{\exists}[\Re]$, an automaton recognizing the relation defined by $\phi$ can be computed

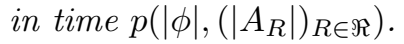

Bounded Valuedness. Let $R \subseteq T_{\Sigma_{1}} \times T_{\Sigma_{2}}$ be a recognizable binary relation. For every $s_{1} \in T_{\Sigma_{1}}$, the number $\# R\left(s_{1}\right)=\left|\left\{s_{2} \mid\left(s_{1}, s_{2}\right) \in R\right\}\right|$ counts the trees in $T_{\Sigma_{2}}$ in relation to it. The valuedness of $R$ is the maximal such number $\operatorname{val}(R)=\max _{s \in T_{\Sigma_{1}}} \# R(s)$. We call $R$ bounded if $\operatorname{val}(R) \leq k$ for some $k \geq 0$.

Lemma 4. A relation $R$ between unranked trees is recognizable iff the corresponding relation between binary trees $f c n s(R)$ is, and $\operatorname{val}(f c n s(R))=\operatorname{val}(R)$.

Theorem 4. For every automaton A recognizing a binary relation $R$ between unranked trees, i.e., $L(A)=\operatorname{ovl}(R)$ :

1. $\operatorname{val}(R)<\infty$ can be decided in P-time in $|A|$.

2. $\operatorname{val}(R)<k$ (for a fixed $k$ ) can be decided in NP-time in $|A|$.

Proof. It is sufficient to prove the theorem for TAs over binary trees by Lemma 4. We start with recognizable relabeling relations, and lift the result to recognizable relations in the long version of the paper. A relabeling relation $R \subseteq$ $T_{\Sigma^{1}} \times \ldots \times T_{\Sigma^{n}}$ is a relation between trees of the same structure, i.e., whenever $\left(s_{1}, \ldots, s_{n}\right) \in R$ then $\operatorname{nod}\left(s_{1}\right)=\ldots=\operatorname{nod}\left(s_{n}\right)$. In other words, the overlays in ovl $(R)$ do not contain any place holder $\perp$.

So let $R \subseteq T_{\Sigma} \times T_{\Delta}$ be a relabeling relation for binary signatures, and $A$ a TA for trees in $T_{\Sigma \times \Delta}$ that recognizes $R$, i.e., $L^{b i n}(A)=\operatorname{ovl}(R)$. We transform 
$A$ into a bottom-up tree transducer $T$ for defining the relation $R$ as in [22]. The rules of $T$ are infered as follows where $x_{1}, x_{2}$ are variables:

$$
\frac{(f, g)\left(p_{1}, p_{2}\right) \rightarrow p \in \operatorname{rul}(A)}{f\left(p_{1}\left(x_{1}\right), p_{2}\left(x_{2}\right)\right) \rightarrow p\left(g\left(x_{1}, x_{2}\right)\right) \in \operatorname{rul}(T)} \quad \frac{(a, b) \rightarrow p \in \operatorname{rul}(A)}{a \rightarrow p(b) \in \operatorname{rul}(T)}
$$

This transducer $T$ has the same valuedness as $R$. Theorem 2.8 of [22] shows that it can be decided in polynomial time whether $T$ is finite-valued, i.e., whether $R$ is bounded. Concerning $k$-valuedness, it can be decided in non-deterministic polynomial time according to Theorem 2.2 of [22].

The polynomials for testing bounded valuedness of tree transducers are much higher than for testing bounded ambiguity for tree automata [23].

Using the above constructions and Theorem 2.7 of [22], we can build an algorithm for computing the exact value of $\operatorname{val}(R)$, if it exists. We can proceed by dichotomy, starting from $2^{2^{P(|A|)}}$, for a fixed polynomial $P$.

From Proposition 4, we get in P-time a non-deterministic automaton recognizing a relation defined by an $F O_{\exists}[\Re]$ formula, and then apply Theorem 4 :

Corollary 1. Let $\Re$ be a finite set of relations and $A_{R}$ deterministic automata recognizing $R \in \Re$. Then there exists a polynomial $p$ such that for formulas $\phi$ in $\mathrm{FO}_{\exists}[\Re]$, the bounded valuedness $\operatorname{val}\left(R_{\phi}\right)<\infty$ of the relation $R_{\phi}$ defined by $\phi$

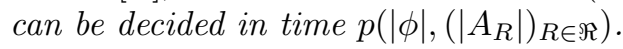

\section{Deciding Bounded Delay and Concurrency}

We prove the main Theorem 3 on deciding bounded delay and concurrency by reduction to Corollary 1 on recognizable relations.

Let $Q$ be an $n$-ary query for trees in $T_{\Sigma}$ and $S \subseteq T_{\Sigma}$ a schema. We define a relation $\operatorname{Can}_{Q}=\left\{\left(t, \operatorname{ren}^{\tau}(s)\right) \mid t * \tau \in \operatorname{can}_{Q} \wedge E q\left(t, s\right.\right.$, ren $\left.\left.{ }^{\text {latest }(\tau)}(t)\right)\right\}$, where $r e n^{\tau}(s)$ is the projection of $s * \tau$ to $\mathbb{B}^{n}$. The relation $B e f=\left\{\left(t, r e n^{\tau}(t), r e n^{e}(t)\right) \mid\right.$ $\left.\tau \in \operatorname{nod}\left(t^{\leq e}\right)^{n}\right\}$ is recognizable by a dTA of size $O\left(2^{n}\right)$, so we cannot use this relation for P-time algorithms without fixing $n$. By using the relation $B e f \mathscr{G} C a n_{Q}=$ $\left\{\left(t, s_{\tau}, s_{e}\right) \mid \operatorname{Can}_{Q}\left(t, s_{\tau}\right)\right.$ and $\left.\operatorname{Bef}\left(t, s_{\tau}, s_{e}\right)\right\}$, the problem can sometimes be circumvented. Given a deterministic automaton defining $Q$ (it can be a TA on fcns encoding, a stepwise tree automaton or an STA), one can construct an automaton of polynomial size recognizing the relation $\mathrm{BefEC} \mathrm{Can}_{Q}$.

Our objective is to define the formulas delay ${ }_{Q}^{S}$ and concur $_{Q}^{S}$ in the logic $F O_{\exists}\left(E q, C a n_{Q}, S, B e f, B e f \mathcal{E} C a n_{Q}\right)$, preferably without using Bef. We start with defining relation $\operatorname{Sel}_{Q}^{S}=\left\{\left(t, \operatorname{ren}^{\tau}(t), \operatorname{ren}^{e}(t)\right) \mid(\tau, e) \in \operatorname{sel}_{Q}^{S}(t)\right\}$ by an FO formula $\operatorname{Sel}_{Q}^{S}\left(X_{t}, X_{\tau}, X_{e}\right)$ with three free variables:

$$
\begin{aligned}
& \operatorname{Sel}_{Q}^{S}\left(X_{t}, X_{\tau}, X_{e}\right)={ }_{d f} S\left(X_{t}\right) \wedge \operatorname{Bef}\left(X_{t}, X_{\tau}, X_{e}\right) \\
& \wedge \forall X_{t}^{\prime} \in T_{\Sigma} \cdot\left(S\left(X_{t}^{\prime}\right) \wedge E q\left(X_{t}, X_{t}^{\prime}, X_{e}\right)\right) \Rightarrow \operatorname{Can}_{Q}\left(X_{t}^{\prime}, X_{\tau}\right)
\end{aligned}
$$

Given automata defining $Q$ and schema $S$, we can thus define an automaton recognizing $\operatorname{Sel}_{Q}^{S}\left(X_{t}, X_{\tau}, X_{e}\right)$. This yields an algorithm for deciding judgments 
$(\tau, e) \in \operatorname{sel}_{Q}^{S}(t)$. It may be unefficient, though, since the automaton obtained this way may be huge, given that formula $\operatorname{Sel}_{Q}^{S}\left(X_{t}, X_{\tau}, X_{e}\right)$ uses full FO-logic of recognizable relations without restriction to some $F O_{\exists}$.

Bounded Delay. We define the relation Delay $Q_{Q}^{S}=\left\{\left(t, \operatorname{ren}^{\tau}(t), \operatorname{ren}^{e}(t)\right) \mid e \in\right.$ $\left.\operatorname{delay}_{Q}^{S}(t, \tau)\right\}$ by the following formula of $F O_{\exists}\left(E q, B e f \in \mathcal{C} C a n_{Q}, S\right)$ :

$$
\begin{gathered}
\operatorname{Delay}_{Q}^{S}\left(X_{t}, X_{\tau}, X_{e}\right)={ }_{d f} \exists X_{t}^{\prime} \in T_{\Sigma} \cdot S\left(X_{t}\right) \wedge \operatorname{BefECan}_{Q}\left(X_{t}, X_{\tau}, X_{e}\right) \\
\wedge S\left(X_{t}^{\prime}\right) \wedge E q\left(X_{t}, X_{t}^{\prime}, X_{e}\right) \wedge \neg \operatorname{Can}_{Q}\left(X_{t}^{\prime}, X_{\tau}\right)
\end{gathered}
$$

All base relations can be defined by deterministic automata of polynomial size when leaving $n$ variable (since we don't need relaton $B e f$ here). Given deterministic automata $A$ and $B$ defining query $Q$ and schema $S=L(B)$, we can thus define a possibly nondeterministic automaton recognizing Delay $S_{Q}\left(X_{t}, X_{\tau}, X_{e}\right)$ in P-time from $A$ and B. Let 2 Delay $_{Q}^{S}=\left\{\left(t \circledast s_{\tau}, s_{e}\right) \mid\right.$ Delay $\left._{Q}^{S}\left(t, s_{\tau}, s_{e}\right)\right\}$. Both relations are recognized by the same automaton. This relation exactly captures the delay: $\operatorname{val}(2 \operatorname{Delay} S)=\left|\operatorname{delay}_{Q}^{S}\right|$. Applying Corollary 1 to $2 \operatorname{Delay}_{Q}^{S}$ proves that bounded delay is decidable in P-time.

Bounded Concurrency. For concurrency, we proceed in a similar manner. The relation Alive $_{Q}^{S}=\left\{\left(t, \operatorname{ren}^{\tau}(t), \operatorname{ren}^{e}(t)\right) \mid \tau \in \operatorname{alive}_{Q}^{S}(t, e)\right\}$ can be defined by the following formula of $F O_{\exists}$ :

$$
\begin{aligned}
& \operatorname{Alive}_{Q}^{S}\left(X_{t}, X_{e}, X_{\tau}\right)=\exists X_{t}^{\prime} \in T_{\Sigma} \cdot \exists X_{t}^{\prime \prime} \in T_{\Sigma} \cdot \exists X_{\tau}^{\prime} \in T_{\mathbb{B}^{n}} . \exists X_{\tau}^{\prime \prime} \in T_{\mathbb{B}^{n}} . S\left(X_{t}^{\prime}\right) \wedge S\left(X_{t}^{\prime \prime}\right) \\
& \quad \wedge \operatorname{Can}_{Q}\left(X_{t}^{\prime}, X_{\tau}^{\prime}\right) \wedge E q_{\Sigma}\left(X_{t}, X_{t}^{\prime}, X_{e}\right) \wedge E q_{\mathbb{B}^{n}}\left(X_{\tau}, X_{\tau}^{\prime}, X_{e}\right) \wedge B e f \cdot\left(X_{\tau}, X_{e}\right) \\
& \quad \wedge \neg \operatorname{Can}_{Q}\left(X_{t}^{\prime \prime}, X_{\tau}^{\prime \prime}\right) \wedge E q_{\Sigma}\left(X_{t}, X_{t}^{\prime \prime}, X_{e}\right) \wedge E q_{\mathbb{B}^{n}}\left(X_{\tau}, X_{\tau}^{\prime \prime}, X_{e}\right) \wedge C_{\mathbb{B}^{n}}\left(X_{\tau}^{\prime \prime}\right)
\end{aligned}
$$

Here, Bef . is like Bef but for partial tuples, and $C_{\mathbb{B}^{n}} \subseteq T_{\mathbb{B}^{n}}$ is the set of trees of type $1^{n}$. Let 2 Alive $Q_{Q}^{S}$ be the binary version of $\operatorname{Alive}_{Q}^{\bar{S}}$, then $\operatorname{val}\left(2 \operatorname{Alive} e_{Q}^{S}\right)=$ $\left|\operatorname{concur}_{Q}^{S}\right|$. Automata for $C_{\mathbb{B}^{n}}$ and $E q_{\mathbb{B}^{n}}$ are necessarily of size $O\left(2^{n}\right)$, which cannot be avoided by embedding them inside other relation like $\neg \mathrm{Can}_{Q}$. But for a fixed $n$, all these automata can be computed in P-time, so that Corollary 1 applied to 2 Alive $S_{Q}^{S}$ proves that bounded concurrency can be decided in P-time.

Conclusion. In this paper we proved that bounded delay and (for fixed $n$ ) bounded concurrency are both computable in P-time, for queries defined by dSTAs. This was obtained by studying some properties of recognizable relations on unranked trees, and combining them with prior results on the valuedness of tree transducers [17]. Considering the P-time translation of a fragment of XPath to dSTAs proposed in [10], we get the same complexity results for this fragment of XPath.

Some questions are left open in the present paper. For fixed $k$ and $n$, deciding $k$-boundedness for delay and concurrency for $n$-ary queries defined by dSTAs is known to be in NP-time. However, NP-hardness is still open. We also chose to define the delay for selection from the time point where the tuple gets complete. An alternative could be to define the $i$ th delay, that starts when $i$ components of the tuple are filled.

Acknowledgments. This work was partially supported by the Enumeration project ANR-07-blanc. 


\section{References}

1. Segoufin, L., Vianu, V.: Validating streaming XML documents. In: ACM PODS. (2002) 53-64

2. Martens, W., Neven, F., Schwentick, T., Bex, G.J.: Expressiveness and complexity of XML schema. ACM Transactions of Database Systems 31(3) (2006) 770-813

3. Gupta, A.K., Suciu, D.: Stream processing of XPath queries with predicates. In: ACM SIGMOD. (2003) 419-430

4. Fernandez, M., Michiels, P., Siméon, J., Stark, M.: XQuery streaming à la carte. In: 23nd International Conference on Data Engineering. (2007) 256-265

5. Benedikt, M., Jeffrey, A.: Efficient and expressive tree filters. In: Foundations of Software Technology and Theoretical Computer Science. LNCS (2007) 461-472

6. Bar-Yossef, Z., Fontoura, M., Josifovski, V.: Buffering in query evaluation over XML streams. In: ACM PODS. (2005) 216-227

7. Olteanu, D.: SPEX: Streamed and progressive evaluation of XPath. IEEE Trans. on Know. Data Eng. 19(7) (2007) 934-949

8. Gou, G., Chirkova, R.: Efficient algorithms for evaluating XPath over streams. In: ACM SIGMOD. (2007) 269-280

9. Berlea, A.: Online evaluation of regular tree queries. Nordic Journal of Computing 13(4) (2006) 1-26

10. Gauwin, O., Niehren, J., Tison, S.: Earliest query answering for deterministic streaming tree automata and a fragment of XPath (2009)

11. Gauwin, O., Niehren, J., Roos, Y.: Streaming tree automata. Information Processing Letters 109(1) (2008) 13-17

12. Alur, R.: Marrying words and trees. In: ACM PODS. (2007) 233-242

13. Alur, R., Madhusudan, P.: Visibly pushdown languages. In: 36th ACM Symposium on Theory of Computing. (2004) 202-211

14. Neumann, A., Seidl, H.: Locating matches of tree patterns in forests. In: Foundations of Software Technology and Theoretical Computer Science. (1998) 134-145

15. Comon, H., Dauchet, M., Gilleron, R., Löding, C., Jacquemard, F., Lugiez, D., Tison, S., Tommasi, M. Available online since 1997: http://tata.gforge.inria. fr Revised October, 12th 2007.

16. Carme, J., Niehren, J., Tommasi, M.: Querying unranked trees with stepwise tree automata. In: 19th International Conference on Rewriting Techniques and Applications. Volume 3091 of LNCS. (2004) 105-118

17. Seidl, H.: Single-valuedness of tree transducers is decidable in polynomial time. Theoretical Computer Science 106 (1992) 135-181

18. Stearns, R.E., Hunt III, H.B.: On the equivalence and containment problems for unambiguous regular expressions, regular grammars and finite automata. SIAM Journal on Computing 14(3) (1985) 598-611

19. Weber, A., Seidl, H.: On the degree of ambiguity of finite automata. In: Mathematical Foundations of Computer Science. Volume 233 of LNCS. (1986) 620-629

20. Allauzen, C., Mohri, M., Rastogi, A.: General algorithms for testing the ambiguity of finite automata. In: DLT. Volume 5257 of LNCS. (2008) 108-120

21. Carme, J., Lemay, A., Niehren, J.: Learning node selecting tree transducer from completely annotated examples. In: 7th International Colloquium on Grammatical Inference. Volume 3264 of Lecture Notes in Artificial Intelligence. (2004) 91-102

22. Seidl, H.: Ambiguity, valuedness and costs (1992) Habilitation Thesis.

23. Sakarovitch, J., de Souza, R.: Decidability of bounded valuedness for transducers. In: Mathematical Foundations of Computer Science. (2008) 